\title{
TWISTED PLANES
}

\author{
JORGE A. GUCCIONE, JUAN J. GUCCIONE, AND CHRISTIAN VALQUI
}

\begin{abstract}
Let $k$ be a commutative ring. We find and characterize a new family of twisted planes (i. e. associative unitary $k$-algebra structures on the $k$-module $k[X, Y]$, having $k[X]$ and $k[Y]$ as subalgebras). Similar results are obtained for the $k$-module of two variables power series $k[[X, Y]]$.
\end{abstract}

\section{INTRODUCTION}

Let $k$ be a commutative ring and let $A, B$ be unitary $k$-algebras. By definition, a twisted tensor product of $A$ with $B$ (over $k$ ) is an algebra structure defined on $A \otimes_{k} B$, with unit $1 \otimes_{k} 1$, such that the canonical maps $i_{A}: A \rightarrow A \otimes_{k} B$ and $i_{B}: B \rightarrow A \otimes_{k} B$ are algebra maps satisfying $a \otimes b=i_{A}(a) i_{B}(b)$. This structure has been formerly studied by many people with different motivations (see for instance [Ca], [C-S-V], [G-G], [Ma], [Tam], [VD-VK]). On one hand it is the most general solution to the problem of factorization of structures in the setting of associative algebras. Consequently, a number of examples of classical and recently defined constructions in ring theory fits into this construction. For instance, Ore extensions, skew group algebras, smash products, etcetera (for the definition and properties of these structures we refer to $[\mathrm{Mo}]$ and $[\mathrm{Ka}])$. On the other hand it has been proposed as the natural representative for the cartesian product of noncommutative spaces, this being based on the existing duality between the categories of algebraic affine spaces and commutative algebras, under which the cartesian product of spaces corresponds to the tensor product of algebras. And last, but not least, twisted tensor products arise as a tool for building algebras starting with simpler ones.

Given algebras $A$ and $B$, a basic problem is to determine all the twisted tensor products of $A$ with $B$ and classify them up to a natural equivalence relation. A (noncommutative) polynomial extension of a $k$-algebra $B$ is a twisted tensor product of a polynomial ring with $B$. A twisted plane is such an extension in which $B$ is also a polynomial algebra $k[X]$. That is, an associative unitary algebra $C$, with underlying $k$-module $k[X, Y]$, such that:

- the natural inclusions $i_{k[X]}: k[X] \rightarrow C$ and $i_{k[Y]}: k[Y] \rightarrow C$ are algebra maps,

- $i_{k[X]}\left(X^{m}\right) i_{k[Y]}\left(Y^{n}\right)=X^{m} Y^{n}$ for each $n, m \geq 0$.

For instance, Ore extensions of $k[X]$ are examples of twisted planes. The aim of this paper is to begin the study of the polynomial extensions, with emphasis in the problem of the classification of the twisted planes. Actually, we do not solve completely this problem in the present work, but we give the first step on having found a new family of twisted planes. Besides the twisted polynomial extensions, in this article we also consider twisted extensions of the power series ring $k[[X]]$, finding a new

2000 Mathematics Subject Classification. Primary 16S10; Secondary 16S80.

Key words and phrases. Twisting maps, Polynomial rings.

Supported by UBACYT 0294 and PIP 5617 (CONICET).

Supported by UBACYT 0294 and PIP 5617 (CONICET).

Supported by PUCP-DAI-3490. 
family of twisted tensor products of $k[[X]]$ with $k[[Y]]$. Indeed, the natural setting to deal with adelically complete algebras such as $k[[X]]$ is the monoidal category of filtered $k$-modules which are complete with respect to the induced topology. Consequently, in this case we look for adelically complete algebras $C$, with underlying topological $k$-module $k[[X, Y]]$, such that:

- the natural inclusions $i_{k[[X]]}: k[[X]] \rightarrow C$ and $i_{k[[Y]]}: k[[Y]] \rightarrow C$ are continuous algebra maps,

- $i_{k[[X]]}\left(X^{m}\right) i_{k[[Y]]}\left(Y^{n}\right)=X^{m} Y^{n}$ for each $n, m \geq 0$.

From now on we assume implicitly that all the maps are $k$-linear maps, all the algebras are over $k$, and the tensor product over $k$ is denoted $\otimes$, without any subscript.

The paper is organized as follows: In Section 1 we have compiled without proofs some of the standard facts on twisted tensor products, thus making our exposition self-contained. In particular we recall the definition of a twisting map $s: A \otimes B \rightarrow B \otimes A$ and we establish the bijective correspondence $s \mapsto B \otimes_{s} A$ between twisting maps and twisted tensor products. We also set up notation and terminology. In Section 2 we begin the study of the noncommutative polynomial extensions. Consider an algebra $A$ and maps $\alpha_{j}: A \rightarrow A(j \geq 0)$. In Theorem 2.1, we determine necessary and sufficient conditions for the existence of a (necessarily unique) twisting map $s: k[Y] \otimes A \rightarrow A \otimes k[Y]$ such that

$$
s(Y \otimes a)=\sum_{j=0}^{\infty} \alpha_{j}(a) \otimes Y^{j} .
$$

When $\alpha_{j}=0$ for all $j \geq 2$, then we reobtain the familiar conditions to build an Ore extension of $A$. That is, $\alpha_{1}$ must be an algebra endomorphism and $\alpha_{0}$ must be an $\left(\alpha_{1}\right.$, id)-derivation. After that we give several examples, and later on, in Theorem 2.7 and Corollary 2.8, we establish a method to construct a twisting map with $\alpha_{0}=0$ and $\alpha_{1}=$ id beginning with a locally nilpotent derivation. Section 3 is devoted to the study of twisted planes. Theorem 3.1 and 3.4 are two of the main results of this paper. Applying them, in Corollary 3.6 we obtain all the twisting maps

$$
s: k[Y] \otimes k[X] \rightarrow k[X] \otimes k[Y]
$$

such that $\alpha_{0}=0, \alpha_{1}$ is the evaluation at an element of $k$ and $\left\{n: \alpha_{n} \neq 0\right\}$ is finite. The aim of Section 4 is to determine all the twisting maps

$$
s: k[Y] \otimes k[t] /\left\langle t^{2}\right\rangle \rightarrow k[t] /\left\langle t^{2}\right\rangle \otimes k[Y] .
$$

To do this we first study the twisted tensor products $k[t] /\left\langle t^{2}\right\rangle \otimes_{s} A$, then we consider in detail the case $A=k[Y]$, and use that $s$ is a twisting map if and only if $\tau \circ{ }^{\circ} \tau$ is, where $\tau$ denotes the flip. Finally, in Section 5 we begin the study of the twisted tensor products of the power series ring $k[[Y]]$ with an algebra $A$, in the monoidal category of complete filtered $k$-modules. In this case, each map

$$
s: k[[Y]] \hat{\otimes} A \rightarrow A \hat{\otimes} k[[Y]]
$$

(where $\hat{\otimes}$ denotes the completed tensor product over $k$ ) is also determined by a family of maps $\alpha_{j}: A \rightarrow A(j \geq 0)$, but the conditions that these maps must satisfy to guarantee that $s$ is a twisting map, which are found in Theorem 5.3, are somewhat different from those required when dealing with noncommutative polynomial extensions. In Theorem 5.4 we give a version for complete algebras of Theorem 2.7, but the main result of this section, and one of the main results of the paper, is Theorem 5.6, in which we obtain all the twisting maps

$$
s: k[[Y]] \hat{\otimes} A \rightarrow A \hat{\otimes} k[[Y]]
$$


with $\alpha_{0}=0$.

Acknowledgment. This research was began during a visit of the first two authors to the "IMCA" and the "PUCP". They specially thank to these institutions for their hospitality and support during their visit. We also thank Professor Guillermo Cortiñas for useful comments.

Acknowledgment. We would like to thank the referee for useful comments and for a very short proof of Theorem 3.4, reducing the original length from eight to less than two pages.

\section{Preliminaries}

In this section we review some of the basic facts about twisted tensor products. For their proofs we refer to [C-S-V], [VD-VK] and [C-I-M-Z]. Given an algebra $A$ we let $\eta_{A}$ and $\mu_{A}$ denote the unit and the multiplication maps of $A$, respectively.

Let $A$ and $B$ be algebras. A twisted tensor product of $A$ with $B$ is an algebra structure on the $k$-module $A \otimes B$, such that the canonical maps

$$
i_{A}: A \rightarrow A \otimes B \quad \text { and } \quad i_{B}: B \rightarrow A \otimes B
$$

are algebra homomorphisms and $\mu \circ\left(i_{A} \otimes i_{B}\right)=\mathrm{id}_{A \otimes B}$, where $\mu$ denotes the multiplication map of the twisted tensor product.

Assume we have a tensor product of $A$ with $B$. Then, the map

$$
s: B \otimes A \rightarrow A \otimes B,
$$

define by $s:=\mu^{\circ}\left(i_{B} \otimes i_{A}\right)$, satisfies:

(1) $s \circ\left(\eta_{B} \otimes A\right)=A \otimes \eta_{B}$ and $s \circ\left(B \otimes \eta_{A}\right)=\eta_{A} \otimes B$,

(2) $s \circ\left(\mu_{B} \otimes A\right)=\left(A \otimes \mu_{B}\right) \circ(s \otimes B) \circ(B \otimes s)$,

(3) $s \circ\left(B \otimes \mu_{A}\right)=\left(\mu_{A} \otimes B\right) \circ(A \otimes s) \circ(s \otimes A)$.

A map satisfying these conditions is called a twisting map. Conversely, if

$$
s: B \otimes A \rightarrow A \otimes B
$$

is a twisting map, then $A \otimes B$ becomes a twisted tensor product via

$$
\mu_{s}:=\left(\mu_{A} \otimes \mu_{B}\right) \circ(A \otimes s \otimes B) .
$$

This algebra will be denoted $A \otimes_{s} B$. Furthermore, these constructions are inverse one of each other.

The twisted tensor product $A \otimes_{s} B$ has the following universal property: Given algebra maps $f: A \rightarrow C$ and $g: B \rightarrow C$ such that

$$
\mu_{C} \circ(g \otimes f)=\mu_{C} \circ(f \otimes g) \circ s,
$$

there is a unique morphism of algebras $h: A \otimes_{s} B \rightarrow C$ satisfying

$$
f=h \circ i_{A} \quad \text { and } \quad g=h \circ i_{B} .
$$

Indeed, it is easy to check that $h=\mu_{C^{\circ}}(f \otimes g)$.

The twisting maps are the objects of a category. Let $s: B \otimes A \rightarrow A \otimes B$ and $t: D \otimes C \rightarrow C \otimes D$ be twisting maps. A morphism $(f, g): s \rightarrow t$ is a pair of morphism of algebras $f: A \rightarrow C$ and $g: B \rightarrow D$ such that

$$
t \circ(g \otimes f)=(f \otimes g) \circ s .
$$

The composition is the evident one. Two twisting maps $s, t: B \otimes A \rightarrow A \otimes B$ are said to be equivalent if they are isomorphic. That is, if there exist automorphisms $f: A \rightarrow A$ and $g: B \rightarrow B$ such that $t=\left(f^{-1} \otimes g^{-1}\right) \circ s^{\circ}(g \otimes f)$. 
The following result is useful to check that a map $s: B \otimes A \rightarrow A \otimes B$ is a twisting map, and will be used implicitly in this paper.

Proposition 1.1. Let $s: B \otimes A \rightarrow A \otimes B$ be a map satisfying conditions (1) and (2). If $\left(b_{i}\right)_{i \in I}$ generates $B$ as an algebra and

$$
s\left(b_{i} \otimes a a^{\prime}\right)=\left(\mu_{A} \otimes B\right) \circ(A \otimes s) \circ(s \otimes A)\left(b_{i} \otimes a \otimes a^{\prime}\right)
$$

for all $a, a^{\prime} \in A$ and each index $i$, then $s$ is a twisting map.

In the last section of this paper we will consider twisting maps between complete filtered algebras. Hence, we will work in the monoidal category CMod, of complete filtered $k$-modules, where $k$ is a commutative ring. An object of CMod is a $k$-module $M$ endowed with a filtration

$$
M=M_{0} \supseteq M_{1} \supseteq M_{2} \supseteq \cdots,
$$

such that each $M_{i}$ is a $k$-module and $M$ is complete with respect to the topology induced by the filtration. A morphism in CMod is a continuous map $f: M \rightarrow N$ (namely, a map $f$ satisfying the requirement that for each $i \geq 0$ there exists $n_{i}$ such that $\left.f\left(M_{n_{i}}\right) \subseteq N_{i}\right)$. The tensor product of $M$ with $N$ in CMod, denoted by $M \hat{\otimes} N$, is the completation of the usual tensor product $M \otimes N$, with respect to the topology induced by the filtration

$$
(M \otimes N)_{i}=\sum_{r+s=i} \jmath\left(M_{r} \otimes N_{s}\right)
$$

where $\jmath: M_{r} \otimes N_{s} \rightarrow M \otimes N$ is the canonical map.

Standard modules are considered as objects of CMod via the filtration

$$
M=M_{0} \supseteq 0=0=\cdots \quad\left(M_{i}=0 \text { for all } i>0\right) .
$$

The power series ring $k[[Y]]$ is an algebra in CMod via the usual filtration

$$
K[[Y]] \supseteq Y k[[Y]] \supseteq Y^{2} k[[Y]] \supseteq \cdots .
$$

Moreover, the completed tensor product $k[[Y]] \hat{\otimes} M$ is canonically isomorphic to $M[[Y]]$, for each standard module $M$.

All the discussion preceding Proposition 1.1 is valid for arbitrary monoidal categories.

\section{Non-COMmutative POLYNOMial EXTENSIONS}

This section is devoted to the study of the twisting maps $k[Y] \otimes A \rightarrow A \otimes k[Y]$, where $A$ is an arbitrary algebra. Given a family of maps $\left(\alpha_{j}: A \rightarrow A\right)_{j \geq 0}$ and indices $n_{1}, \ldots, n_{r} \geq 0$, we set $\left|n_{1}, \ldots, n_{r}\right|=n_{1}+\cdots+n_{r}$ and $\alpha_{n_{1} \ldots n_{r}}=\alpha_{n_{1}}{ } \cdots \circ \alpha_{n_{r}}$. Moreover we write

$$
\gamma_{j}^{(0)}=\delta_{0 j} \text { id } \quad \text { and } \quad \gamma_{j}^{(r)}=\sum_{\left|n_{1}, \ldots, n_{r}\right|=j} \alpha_{n_{1} \ldots n_{r}} \text { for } r>0,
$$

where $\delta_{0 j}$ denotes the symbol of Kronecker. Note that $\gamma_{j}^{(1)}=\alpha_{j}$.

Theorem 2.1. Let $A$ be an algebra and $s: k[Y] \otimes A \rightarrow A \otimes k[Y]$ a twisting map. The equation

$$
s(Y \otimes a)=\sum_{j=0}^{\infty} \alpha_{j}(a) \otimes Y^{j},
$$

defines a family of maps $\alpha_{j}: A \rightarrow A$, which satisfies:

(1) For each $a \in A$ there exists $j_{0} \geq 0$, such that $\alpha_{j}(a)=0$ whenever $j>j_{0}$.

(2) $\alpha_{j}(1)=\delta_{j 1}$. 
(3) For all $j \geq 0$ and all $a, b \in A$,

$$
\alpha_{j}(a b)=\sum_{r=0}^{\infty} \alpha_{r}(a) \gamma_{j}^{(r)}(b)
$$

Moreover,

$$
s\left(Y^{r} \otimes a\right)=\sum_{j=0}^{\infty} \gamma_{j}^{(r)}(a) \otimes Y^{j}
$$

for all $r \geq 0$ and $a \in A$. Conversely, given maps $\alpha_{j}: A \rightarrow A(j \geq 0)$ satisfying (1)-(3), the formula (2.2) defines a twisting map.

Proof. Let $s$ be a twisting map. The formula for $s\left(Y^{r} \otimes a\right)$ can be easily checked by induction on $r$, using that $s(1 \otimes a)=a \otimes 1$ and the compatibility of $s$ with the multiplication of $k[Y]$. Item (1) is immediate and items (2) and (3) are consequences of the compatibility of $s$ with the unit and the multiplication of $A$. Conversely, assume we have a family of maps $\left(\alpha_{j}\right)_{j \geq 0}$ satisfying (1), (2) and (3). Let

$$
f: A[Y] \rightarrow A[Y]
$$

be the map given by $f\left(a Y^{j}\right)=\sum_{i=0}^{\infty} \alpha_{i}(a) Y^{i+j}$, which is well defined by (1). Since $f^{r}(a)=\sum_{j=0}^{\infty} \gamma_{j}^{(r)}(a) Y^{j}$ and obviously $f^{r}(a) \in A[Y]$, for each $a \in A$ and $r>0$, there exists $n \geq 0$ such that $\gamma_{j}^{(r)}(a)=0$ whenever $j>n$. This establishes the well-definition of formula (2.2). We leave the proof that $s$ is a twisting map to the reader.

Remark 2.2. If $s: k[Y] \otimes A \rightarrow A \otimes k[Y]$ is a twisting map, then $\operatorname{ker}\left(\alpha_{0}\right)$ is a subalgebra of $A$. Moreover,

- If $\alpha_{0}=0$, then $\alpha_{1}$ is a endomorphism of algebras.

- Let $\nu>1$. If $\alpha_{0}=0$ and $\alpha_{j}=0$ for $1<j<\nu$, then

$$
\alpha_{\nu}(a b)=\alpha_{1}(a) \alpha_{\nu}(b)+\alpha_{\nu}(a) \alpha_{1}^{\nu}(b) .
$$

Example 2.3. If $\alpha: A \rightarrow A$ is an algebra endomorphism and $\delta: A \rightarrow A$ is an $\alpha$-derivation (that is $\delta(a b)=\delta(a) b+\alpha(a) \delta(b))$, then there is a unique twisting map $s: k[Y] \otimes A \rightarrow A \otimes k[Y]$ such that

$$
s(Y \otimes a)=\alpha(a) \otimes Y+\delta(a) \otimes 1 \quad \text { for all } a \in A .
$$

Example 2.4. Let $A=k[t] /\left\langle t^{2}\right\rangle$. Consider the family of maps $\left(\alpha_{j}: A \rightarrow A\right)_{j \geq 0}$, defined by

$$
\alpha_{0}=0, \quad \alpha_{1}=\mathrm{id}, \quad \alpha_{2}(\lambda+\mu t)=\mu t \quad \text { and } \quad \alpha_{j}=0 \text { for } j>2 .
$$

The formula $s(Y \otimes a)=\alpha_{1}(a) \otimes Y+\alpha_{2}(a) \otimes Y^{2}$ defines a twisting map.

Let $\alpha: A \rightarrow A$ be an algebra automorphism and let $\left(\beta_{i}: A \rightarrow A\right)_{i \geq 1}$ be a family of maps. For $i_{1}, \ldots, i_{l} \geq 1$, let

$$
\beta_{\left(i_{1}, \ldots, i_{l}\right)}=\beta_{i_{1}} \circ \alpha^{-1} \circ \beta_{i_{2}} \circ \alpha^{-1} \circ \ldots \circ \beta_{i_{l-2}} \circ \alpha^{-1} \circ \beta_{i_{l-1}} \circ \alpha^{-1} \circ \beta_{i_{l}}
$$

Note that $\beta_{(i)}=\beta_{i}$. If $i_{1}, \ldots, i_{l}=1$ we will write $\beta_{(1)}^{(l)}$ instead of $\beta_{(1, \ldots, 1)}$. In particular $\beta_{(1)}^{(1)}=\beta_{(1)}=\beta_{1}$. We also write $\beta_{(1)}^{(0)}=\alpha$.

Lemma 2.5. Let $\left(\alpha_{j}: A \rightarrow A\right)_{j \geq 0}$ be the family of maps defined by $\alpha_{0}=0, \alpha_{1}=\alpha$ and

$$
\alpha_{j}=\sum_{l=1}^{j-1} \sum_{\left|i_{1}, \ldots, i_{l}\right|=j-1} \beta_{\left(i_{1}, \ldots, i_{l}\right)} \quad \text { for } j \geq 2 .
$$


Then, for all $j \geq r$,

$$
\gamma_{j}^{(r)}=L+\sum_{\substack{n_{1}, \ldots, n_{r} \geq 0 \\\left|n_{1}, \ldots, n_{r}\right|=j-r}} \beta_{(1)}^{\left(n_{1}\right)} \circ \cdots \circ \beta_{(1)}^{\left(n_{r}\right)},
$$

where $L$ is sum of compositions of $\alpha^{\prime}$ 's, $\alpha^{-1}$ 's and $\beta_{i}$ 's, in which at least one $\beta_{i}$ with $i>1$, appears.

Proof. Since

$$
\alpha_{j}=\beta_{1}^{(j-1)}+\sum_{l=1}^{j-2} \sum_{\left|i_{1}, \ldots, i_{l}\right|=j-1} \beta_{\left(i_{1}, \ldots, i_{l}\right)} \quad \text { for all } j \geq 1
$$

we have

$$
\begin{aligned}
\gamma_{j}^{(r)} & =\sum_{\left|n_{1}, \ldots, n_{r}\right|=j} \alpha_{n_{1} \ldots n_{r}} \\
& =L+\sum_{\substack{\left|n_{1}, \ldots, n_{r}\right|=j \\
n_{(1)}}} \beta_{\substack{n_{1}, \ldots, n_{r} \geq 0 \\
\left|n_{1}, \ldots, n_{r}\right|=j-r}}^{\left(n_{1}-1\right)} \beta_{(1)}^{\left(n_{1}\right)} \ldots \ldots \beta_{(1)}^{\left(n_{r}-1\right)}, \\
& =L+\beta_{(1)}^{\left(n_{r}\right)},
\end{aligned}
$$

as desired.

Let $A$ be an algebra and $\varphi, \psi$ endomorphisms of $A$. Recall that a map $d: A \rightarrow A$ is a $(\varphi, \psi)$-derivation if

$$
d(a b)=d(a) \psi(b)+\varphi(a) d(b) \text { for all } a, b \in A .
$$

Lemma 2.6. For each $i \geq 1$, let $\beta_{i}: A \rightarrow A$ be an $\left(\alpha, \alpha^{i+1}\right)$-derivation. Assume that if $i+i^{\prime} \geq 3$, then $\alpha^{r}\left(\beta_{i}(a)\right) \beta_{i^{\prime}}(b)=0$ for all $r \in \mathbb{Z}$ and $a, b \in A$. We have:

(1) $\beta_{\left(i_{1}, \ldots, i_{l}\right)}(a) L(b)=0$, where $L$ is as in Lemma 2.5.

(2) If some $i_{u}>1$ and some $n_{v}>0$, then

$$
\beta_{\left(i_{1}, \ldots, i_{l}\right)}(a) \alpha^{h} \circ \beta_{1}(b)=\alpha^{h} \circ \beta_{1}(a) \beta_{\left(i_{1}, \ldots, i_{l}\right)}(b)=0 .
$$

(3) If some $i_{u}>1$, then

$$
\beta_{\left(i_{1}, \ldots, i_{l}\right)} \text { is an }\left(\alpha, \alpha^{j}\right) \text {-derivation, }
$$

where $j=1+i_{1}+\cdots+i_{l}$.

Proof. (1) let $f_{1} \circ \ldots \circ f_{v}$, where $f_{i}=\left\{\alpha, \alpha^{-1}, \beta_{1}, \beta_{2}, \ldots\right\}$ be a term of $L$. Let $i_{0}$ be the least $i$ such that $f_{i}=\beta_{j}$ with $j>1$. By definition $\beta_{\left(i_{1}, \ldots, i_{l}\right)}(a)=\beta_{i_{1}}\left(a^{\prime}\right)$, where $a^{\prime}=\alpha^{-1}\left(\beta_{\left(i_{2}, \ldots, i_{l}\right)}(a)\right)$. If $i_{0}=1$, then

$$
\beta_{i_{1}}\left(a^{\prime}\right)\left(f_{1} \circ \cdots \circ f_{v}(b)\right)=0,
$$

by hypothesis. The general case follows by induction on $i_{0}$ using that if $f_{1}=\alpha^{ \pm 1}$, then

$$
\beta_{i_{1}}\left(a^{\prime}\right) f_{1} \circ \cdots \circ f_{v}(b)=f_{1}\left(f_{1}^{-1} \circ \beta_{i_{1}}\left(a^{\prime}\right) f_{2} \circ \cdots \circ f_{v}(b)\right)
$$

and if $f_{1}=\beta_{1}$, then

$$
\begin{aligned}
\beta_{i_{1}}\left(a^{\prime}\right) f_{1} \circ \cdots \circ f_{v}(b) & =\beta_{1}\left(\alpha^{-1} \circ \beta_{i_{1}}\left(a^{\prime}\right) f_{2^{\circ}} \cdots \circ f_{v}(b)\right) \\
& +\alpha^{2}\left(\alpha^{-2} \circ \beta_{1} \circ \alpha^{-1} \circ \beta_{i_{1}}\left(a^{\prime}\right) f_{2} \circ \cdots \circ f_{v}(b)\right) .
\end{aligned}
$$

(2) It is similar to (1). 
(3) We make the proof by induction on $l$. First assume that $u>1$. Then, by the inductive hypothesis,

$$
\begin{aligned}
\beta_{\left(i_{1}, \ldots, i_{l}\right)}(a b) & =\beta_{i_{1} \circ \alpha^{-1} \circ} \beta_{\left(i_{2}, \ldots, i_{l}\right)}(a b) \\
& =\beta_{i_{1} \circ} \alpha^{-1}\left(\beta_{\left(i_{2}, \ldots, i_{l}\right)}(a) \alpha^{1+i_{2}+\cdots+i_{l}}(b)+\alpha(a) \beta_{\left(i_{2}, \ldots, i_{l}\right)}(b)\right) \\
& =\beta_{\left(i_{1}, \ldots, i_{l}\right)}(a) \alpha^{1+i_{1}+\cdots+i_{l}}(b)+\beta_{\left(i_{2}, \ldots, i_{l}\right)}(a) \beta_{i_{1}} \circ \alpha^{i_{2}+\cdots+i_{l}}(b) \\
& +\beta_{i_{1}}(a) \alpha^{i_{1}} \circ \beta_{\left(i_{2}, \ldots, i_{l}\right)}(b)+\alpha(a) \beta_{\left(i_{1}, \ldots, i_{l}\right)}(b) \\
& =\beta_{\left(i_{1}, \ldots, i_{l}\right)}(a) \alpha^{1+i_{1}+\cdots+i_{l}}(b)+\alpha(a) \beta_{\left(i_{1}, \ldots, i_{l}\right)}(b),
\end{aligned}
$$

where the last equality follows from the fact that, by item (2),

$$
\beta_{\left(i_{2}, \ldots, i_{l}\right)}(a) \beta_{i_{1}} \circ \alpha^{i_{2}+\cdots+i_{l}}(b)=\beta_{i_{1}}(a) \alpha^{i_{1} \circ} \beta_{\left(i_{2}, \ldots, i_{l}\right)}(b)=0 .
$$

Assume now that $u=1$. Then, arguing as above we obtain,

$$
\begin{aligned}
\beta_{\left(i_{1}, \ldots, i_{l}\right)}(a b) & =\beta_{\left(i_{1}, \ldots, i_{l-1}\right)^{\circ} \alpha^{-1} \circ \beta_{i_{l}}(a b)} \\
& =\beta_{\left(i_{1}, \ldots, i_{l}\right)}(a) \alpha^{1+i_{1}+\cdots+i_{l}}(b)+\alpha(a) \beta_{\left(i_{1}, \ldots, i_{l}\right)}(b),
\end{aligned}
$$

as desired.

Theorem 2.7. Let $\alpha: A \rightarrow A$ be an algebra automorphism. For each $i \geq 1$, let $\beta_{i}: A \rightarrow A$ be an $\left(\alpha, \alpha^{i+1}\right)$-derivation. If

(1) $\alpha^{r}\left(\beta_{i}(a)\right) \beta_{i^{\prime}}(b)=0$ for all $r \in \mathbb{Z}$ and $a, b \in A$ whenever $i+i^{\prime} \geq 3$,

(2) For all $a \in A$ there is $n \in \mathbb{N}$ such that

$$
\sum_{l=1}^{j} \sum_{\left|i_{1}, \ldots, i_{l}\right|=j} \beta_{\left(i_{1}, \ldots, i_{l}\right)}(a)=0 \quad \text { for all } j>n,
$$

then, the formula

$$
s(Y \otimes a)=\sum_{j=0}^{\infty} \alpha_{j}(a) \otimes Y^{j},
$$

where the maps $\alpha_{j}: A \rightarrow A(j \geq 0)$ are constructed as in Lemma 2.5, defines a twisting map s: $k[Y] \otimes A \rightarrow A \otimes k[Y]$.

Proof. By item (2), the maps $\alpha_{j}$ satisfy condition (1) of Theorem 2.1. Condition (2) follows from the fact that the $\beta_{i}$ 's are derivations. It remains to check that condition (3) also holds. For $j \leq 1$ this is immediate. Assume $j \geq 2$ and set

$$
T=\sum_{r=0}^{\infty} \alpha_{r}(a) \gamma_{j}^{(r)}(b) .
$$

On one hand, by Lemma 2.5 and 2.6 ,

$$
\begin{aligned}
T & =\sum_{r=1}^{j} \alpha_{r}(a) \gamma_{j}^{(r)}(b) \\
& =\alpha_{1}(a) \gamma_{j}^{(1)}(b)+\alpha_{j}(a) \gamma_{j}^{(j)}(b)+\sum_{r=2}^{j-1} \alpha_{r}(a) \gamma_{j}^{(r)}(b) \\
& =\sum_{l=1}^{j-1} \sum_{\left|i_{1}, \ldots, i_{l}\right|=j-1} \alpha(a) \beta_{\left(i_{1}, \ldots, i_{l}\right)}(b)+\sum_{l=1}^{j-1} \sum_{\left|i_{1}, \ldots, i_{l}\right|=j-1} \beta_{\left(i_{1}, \ldots, i_{l}\right)}(a) \alpha^{j}(b) \\
& +\sum_{r=2}^{j-1}\left(\sum_{l=1}^{r-1} \sum_{\left|i_{1}, \ldots, i_{l}\right|=r-1} \beta_{\left(i_{1}, \ldots, i_{l}\right)}(a)\right)\left(L(b)+\sum_{\left|n_{1}, \ldots, n_{r}\right|=j-r} \beta_{(1)}^{\left(n_{1}\right)} \ldots \circ \beta_{(1)}^{\left(n_{r}\right)}(b)\right)
\end{aligned}
$$




$$
\begin{aligned}
& =\sum_{l=1}^{j-1} \sum_{\left|i_{1}, \ldots, i_{l}\right|=j-1} \alpha(a) \beta_{\left(i_{1}, \ldots, i_{l}\right)}(b)+\sum_{l=1}^{j-1} \sum_{\left|i_{1}, \ldots, i_{l}\right|=j-1} \beta_{\left(i_{1}, \ldots, i_{l}\right)}(a) \alpha^{j}(b) \\
& +\sum_{r=2}^{j-1} \sum_{\left|n_{1}, \ldots, n_{r}\right|=j-r} \beta_{(1)}^{(r-1)}(a) \beta_{(1)}^{\left(n_{1}\right)} \ldots \ldots \circ \beta_{(1)}^{\left(n_{r}\right)}(b) .
\end{aligned}
$$

On the other hand, since by item (3) of Lemma $2.6, \beta_{\left(i_{1}, \ldots, i_{l}\right)}$ is an $\left(\alpha, \alpha^{j}\right)$-derivation whenever some $i_{u}>1$, we have

$$
\begin{aligned}
\alpha_{j}(a b) & =\sum_{l=1}^{j-1} \sum_{\left|i_{1}, \ldots, i_{l}\right|=j-1} \beta_{\left(i_{1}, \ldots, i_{l}\right)}(a b) \\
& =\sum_{l=1}^{j-2} \sum_{\left|i_{1}, \ldots, i_{l}\right|=j-1}\left(\alpha(a) \beta_{\left(i_{1}, \ldots, i_{l}\right)}(b)+\beta_{\left(i_{1}, \ldots, i_{l}\right)}(a) \alpha^{j}(b)\right)+\beta_{(1)}^{(j-1)}(a b) .
\end{aligned}
$$

So, in order to finish the proof it suffices to show that for all $j \geq 2$,

$$
\begin{aligned}
\beta_{(1)}^{(j-1)}(a b) & =\alpha(a) \beta_{(1)}^{(j-1)}(b)+\beta_{(1)}^{(j-1)}(a) \alpha^{j}(b) \\
& +\sum_{r=2}^{j-1} \sum_{\left|n_{1}, \ldots, n_{r}\right|=j-r} \beta_{(1)}^{(r-1)}(a) \beta_{(1)}^{\left(n_{1}\right)} \ldots \circ \beta_{(1)}^{\left(n_{r}\right)}(b) .
\end{aligned}
$$

We proceed by induction on $j$. When $j=2$,

$$
\beta_{(1)}^{(1)}(a b)=\beta_{1}(a b)=\alpha(a) \beta_{1}(b)+\beta_{1}(a) \alpha^{2}(b),
$$

since $\beta_{1}$ is an $\left(\alpha, \alpha^{2}\right)$-derivation. Assume that the result is valid for $j$. Then,

$$
\begin{aligned}
& \beta_{(1)}^{(j)}(a b)=\beta_{1} \circ \alpha^{-1} \circ \beta_{(1)}^{(j-1)}(a b) \\
& =\beta_{1}\left(a \alpha^{-1} \circ \beta_{(1)}^{(j-1)}(b)\right)+\beta_{1}\left(\alpha^{-1} \beta_{(1)}^{(j-1)}(a) \alpha^{j-1}(b)\right) \\
& +\beta_{1}\left(\sum_{r=2}^{j-1} \sum_{\left|n_{1}, \ldots, n_{r}\right|=j-r} \alpha^{-1 \circ} \beta_{(1)}^{(r-1)}(a) \alpha^{-1} \circ \beta_{(1)}^{\left(n_{1}\right)} \circ \ldots \circ \beta_{(1)}^{\left(n_{r}\right)}(b)\right) \\
& =\alpha(a) \beta_{(1)}^{(j)}(b)+\beta_{(1)}^{(j)}(a) \alpha^{j+1}(b) \\
& +\beta_{1}(a) \alpha^{\circ} \beta_{(1)}^{(j-1)}(b)+\beta_{(1)}^{(j-1)}(a) \beta_{1} \circ \alpha^{j-1}(b) \\
& +\sum_{r=2}^{j-1} \sum_{\left|n_{1}, \ldots, n_{r}\right|=j-r} \beta_{(1)}^{(r)}(a) \alpha \circ \beta_{(1)}^{\left(n_{1}\right)} \ldots \ldots \circ \beta_{(1)}^{\left(n_{r}\right)}(b) \\
& +\sum_{r=2}^{j-1} \sum_{\left|n_{1}, \ldots, n_{r}\right|=j-r} \beta_{(1)}^{(r-1)}(a) \beta_{(1)}^{\left(n_{1}+1\right)} \circ \cdots \circ \beta_{(1)}^{\left(n_{r}\right)}(b) \\
& =\alpha(a) \beta_{(1)}^{(j)}(b)+\beta_{(1)}^{(j)}(a) \alpha^{j+1}(b) \\
& +\sum_{r=2}^{j} \sum_{\left|n_{1}, \ldots, n_{r}\right|=j+1-r} \beta_{(1)}^{(r-1)}(a) \beta_{(1)}^{\left(n_{1}\right)} \ldots \circ \beta_{(1)}^{\left(n_{r}\right)}(b) .
\end{aligned}
$$

This finishes the proof.

Corollary 2.8. Let $A$ be an algebra, $\alpha: A \rightarrow A$ be an algebra automorphism and $\beta: A \rightarrow A$ be an $\left(\alpha, \alpha^{2}\right)$-derivation. Let $\left(\alpha_{j}: A \rightarrow A\right)_{j \geq 0}$ be the family of maps defined by $\alpha_{0}=0$ and $\alpha_{j}=\left(\beta \circ \alpha^{-1}\right)^{j-2} \circ \beta$ for $j \geq 1$. If $\beta \circ \alpha^{-1}$ is locally nilpotent 
(that is, for each $a \in A$ there exists $n \geq 1$ such that $\left.\left(\beta \circ \alpha^{-1}\right)^{n}(a)=0\right)$, then the formula

$$
s(Y \otimes a)=\sum_{j=0}^{\infty} \alpha_{j}(a) \otimes Y^{j}
$$

defines a twisting map $s: k[Y] \otimes A \rightarrow A \otimes k[Y]$.

Proof. Take $\beta_{1}=\beta$ and $\beta_{j}=0$ for $j>1$ in Theorem 2.7.

Example 2.9. Let $A=k[t] /\left\langle t^{n}\right\rangle$. Let $D: A \rightarrow A$ be the derivation defined by $D(P)(t)=P^{\prime}(t) t^{2}$. The formula

$$
s(Y \otimes P)=P \otimes Y+\sum_{j=1}^{\infty} D^{j}(P) \otimes Y^{j+1}
$$

defines a twisting map $s: k[Y] \otimes A \rightarrow A \otimes k[Y]$.

\section{Twisted Planes}

The aim of this section is to study in detail the twisting maps

$$
s: k[Y] \otimes k[X] \rightarrow k[X] \otimes k[Y] .
$$

We use implicitly the canonical identification $k[X] \otimes k[Y] \simeq k[X, Y]$. Consequently, given $Q=\sum_{i j} q_{i j} X^{i} \otimes Y^{j} \in k[Y] \otimes k[X]$, we write

$$
\frac{\partial^{m+n} Q}{\partial X^{m} \partial Y^{n}}(X, Y)=\sum_{i \geq m} \sum_{j \geq n} \frac{i !}{(i-m) !} \frac{j !}{(j-n) !} q_{i j} X^{i-m} Y^{j-n}
$$

for all $m, n \geq 0$.

Theorem 3.1. For each $Q \in X^{2} k[X] \otimes Y^{2} k[Y]$ there is a unique twisting map $s: k[Y] \otimes k[X] \rightarrow k[X] \otimes k[Y]$ such that $s(Y \otimes X)=Q$. Moreover $s\left(Y^{r} \otimes X^{s}\right)=0$ whenever $r, s>0$ and $r+s>2$.

Proof. First we assume that a such twisting map exists, and we prove that it satisfies $s\left(Y^{r} \otimes X^{s}\right)=0$ if $r, s>0$ and $r+s>2$. Let $\left(\alpha_{j}\right)_{j \geq 0}$ be as in Theorem 2.1. So, $\alpha_{j}(X)=\frac{\partial^{j} Q}{\partial Y^{j}}(X, 0)$. The hypothesis means that $\alpha_{0}(X)=\alpha_{1}(X)=0$ and $\alpha_{j}(X) \in X^{2} k[X]$ for each $j \geq 2$. Consequently, by Remark 2.2, $\alpha_{0}=0$ and $\alpha_{1}$ is the evaluation in 0 . We assert that $\alpha_{j}\left(X^{n}\right)=0$ for each $j \geq 0$ and $n \geq 2$. For $j=0,1$ this is clear. Assume $\alpha_{l}\left(X^{n}\right)=0$ for all $l<j$ and $n \geq 2$. Then, by item (3) of Theorem 2.1, we have

$$
\alpha_{j}\left(X^{2}\right)=\sum_{r=2}^{j} \alpha_{r}(X) \gamma_{j}^{(r)}(X)
$$

which vanishes, because clearly $\alpha_{n_{1} \ldots n_{r}}(X)=0$ if $n_{r} \leq 1$, and also if $n_{r} \geq 2$ since, in this case, $\alpha_{n_{r}}(X) \in X^{2} k[X]$ and $n_{r-1}<j$. Assuming now that $n \geq 3$ and $\alpha_{j}\left(X^{n}\right)=0$, using again item (3) of Theorem 2.1, we obtain

$$
\alpha_{j}\left(X^{n}\right)=\sum_{r=2}^{j} \alpha_{r}\left(X^{2}\right) \gamma_{j}^{(r)}\left(X^{n-2}\right)=0 .
$$

It is now easy to check that $s\left(Y^{r} \otimes X^{s}\right)=0$ whenever $r, s>0$ and $r+s>2$, as wanted. Finally, to check the existence of $s$, it suffices to note that the family of maps $\left(\alpha_{j}: A \rightarrow A\right)_{j \geq 0}$, defined by $\alpha_{0}=0, \alpha_{1}\left(X^{n}\right)=\delta_{1 n}$ and

$$
\alpha_{j}\left(X^{n}\right)= \begin{cases}\frac{\partial^{j} Q}{\partial Y^{j}}(X, 0) & \text { if } n=1 \\ 0 & \text { otherwise }\end{cases}
$$


for $j \geq 2$ satisfies the conditions required in Theorem 2.1. We leave the details to the reader.

Definition 3.2. A twisting map $s: k[Y] \otimes k[X] \rightarrow k[X] \otimes k[Y]$ is left bounded if there exists $n_{0} \in \mathbb{N}$ such that

$$
s\left(Y \otimes X^{n}\right) \subseteq k[X] \otimes \bigoplus_{i=0}^{n} k Y^{i} \quad \text { for all } n \geq n_{0} .
$$

It is right bounded if $\tau \circ S^{\circ} \tau$ is left bounded, where $\tau$ denotes the flip. In other words, if there exists $n_{0} \in \mathbb{N}$ such that

$$
s\left(Y^{n} \otimes X\right) \subseteq \bigoplus_{i=0}^{n} k X^{i} \otimes k[Y] \quad \text { for all } n \geq n_{0} .
$$

Finally, we say that $s$ is bounded if it is left and right bounded.

Example 3.3. Twisting maps associated with Ore extensions $k[X][Y, \alpha, \delta]$ are left bounded, but in general they are not right bounded. The twisting maps introduced in Theorem 3.1 are bounded.

By the sake of continuity the proof of the following result is relegated to an appendix.

Theorem 3.4. Assume that $k$ is a commutative domain. Let $Q \in k[X] \otimes k[Y]$. The following facts hold:

(1) If $Q \in k[X] \otimes Y^{2} k[Y]$ and there is a (necessarily unique) left bounded twisting map $s: k[Y] \otimes k[X] \rightarrow k[X] \otimes k[Y]$ such that $s(Y \otimes X)=Q$, then $Q \in X^{2} k[X] \otimes Y^{2} k[Y]$.

(2) If $Q \in X^{2} k[X] \otimes k[Y]$ and there is a (necessarily unique) right bounded twisting map $s: k[Y] \otimes k[X] \rightarrow k[X] \otimes k[Y]$ such that $s(Y \otimes X)=Q$, then $Q \in X^{2} k[X] \otimes Y^{2} k[Y]$.

We say that a twisting map $s: k[Y] \otimes k[X] \rightarrow k[X] \otimes k[Y]$ is almost null if it is equivalent (in the sense introduced above Proposition 1.1) to one of the twisting maps considered in Theorem 3.1.

Corollary 3.5. Assume that $k$ is a commutative domain. Let $R \in k[X] \otimes k[Y]$. The following facts hold:

(1) There is an almost null twisting map $s^{\prime}: k[X] \otimes k[Y] \rightarrow k[Y] \otimes k[X]$ such that $s^{\prime}(Y \otimes X)=R$, if and only if there exist $\lambda, \xi \in k$ satisfying:

(a) $\xi$ is multiple root of $\frac{\partial^{i} R}{\partial X^{i}}(0, Y)$ for each $i>1$,

$$
R(0, \xi)=0, \quad \frac{\partial R}{\partial X}(0, \xi)=\xi, \quad \frac{\partial R}{\partial Y}(0, \xi)=\lambda, \quad \frac{\partial^{2} R}{\partial X \partial Y}(0, \xi)=0
$$

(b) $\lambda$ is multiple root of $\frac{\partial^{j} R}{\partial Y^{j}}(X, 0)$ for each $j>1$,

$$
R(\lambda, 0)=0, \quad \frac{\partial R}{\partial Y}(\lambda, 0)=\lambda, \quad \frac{\partial R}{\partial X}(\lambda, 0)=\xi, \quad \frac{\partial^{2} R}{\partial X \partial Y}(\lambda, 0)=0,
$$

Moreover, the equivalence $s^{\prime} \simeq s$ is realized by means of the automorphisms

$$
f: k[Y] \rightarrow k[Y] \quad \text { and } \quad g: k[X] \rightarrow k[X]
$$

defined by $f(Y)=Y-\xi$ and $g(X)=X-\lambda$.

(2) If there exist $\lambda, \xi \in k$ that satisfy item (a), but not item (b), then there is not a left bounded twisting map $s^{\prime}: k[Y] \otimes k[X] \rightarrow k[X] \otimes k[Y]$ such that $s^{\prime}(Y \otimes X)=R$. 
(3) If there exist $\lambda, \xi \in k$ that satisfy item (b), but not item (a), then there is not a right bounded twisting map $s^{\prime}: k[Y] \otimes k[X] \rightarrow k[X] \otimes k[Y]$ such that $s^{\prime}(Y \otimes X)=R$.

Proof. It is easy to check that $s^{\prime}: k[Y] \otimes k[X] \rightarrow k[X] \otimes k[Y]$ is an almost null twisting map if and only if there exist $\lambda, \xi \in k$ such that

$$
s=\left(g^{-1} \otimes f^{-1}\right) \circ s^{\prime} \circ(f \otimes g)
$$

satisfies the conditions required in Theorem 3.1, where

$$
f: k[Y] \rightarrow k[Y] \text { and } g: k[X] \rightarrow k[X]
$$

are the automorphisms defined by $f(Y)=Y-\xi$ and $g(X)=X-\lambda$. Write

$$
s(Y \otimes X)=\sum_{i j} q_{i j} X^{i} \otimes Y^{j} \quad \text { and } \quad s^{\prime}(Y \otimes X)=\sum_{i j} r_{i j} X^{i} \otimes Y^{j} .
$$

Let $U=R-X \otimes Y$. A direct computation shows that

$$
\begin{aligned}
Q(X, Y) & =s(Y \otimes X) \\
& =\left(\left(g^{-1} \otimes f^{-1}\right) \circ s^{\prime} \circ(f \otimes g)\right)(Y \otimes X) \\
& =\sum_{i j}\left(\sum_{m n=0}^{\infty}\left(\begin{array}{c}
m \\
i
\end{array}\right)\left(\begin{array}{c}
n \\
j
\end{array}\right) \lambda^{m-i} \xi^{n-j} r_{m n}\right) X^{i} \otimes Y^{j}-X \otimes \xi-\lambda \otimes Y-\lambda \otimes \xi, \\
& =\sum_{i j} \frac{1}{i !} \frac{1}{j !} \frac{\partial^{i+j} U}{\partial X^{i} \partial Y^{j}}(\lambda, \xi) X^{i} \otimes Y^{j} \\
& =U(X+\lambda, Y+\xi),
\end{aligned}
$$

where we have adopted the usual convention that a combinatorial number is zero if its numerator is lesser than its denominator. So $s^{\prime}$ is almost null if and only if there is $\lambda, \mu \in k$ such that $U(X+\lambda, Y+\xi) \in X^{2} k[X] \otimes Y^{2} k[Y]$. Note now that $U(X+\lambda, Y+\xi) \in k[X] \otimes Y^{2} k[Y]$ if and only if

$$
\frac{\partial^{i} U}{\partial X^{i}}(\lambda, \xi)=\frac{\partial^{i+1} U}{\partial X^{i} \partial Y}(\lambda, \xi)=0 \quad \text { for all } i \geq 0,
$$

and $U(X+\lambda, Y+\xi) \in X^{2} k[X] \otimes k[Y]$ if and only if

$$
\frac{\partial^{j} U}{\partial Y^{j}}(\lambda, \xi)=\frac{\partial^{1+j} U}{\partial X \partial Y^{j}}(\lambda, \xi)=0 \text { for all } j \geq 0 .
$$

Since

$$
\frac{\partial^{i} U}{\partial X^{i}}(\lambda, \xi)=\sum_{n \geq 0} \frac{\partial^{i+n} U}{\partial X^{i}}(0, \xi) \lambda^{n} \quad \text { and } \quad \frac{\partial^{i} U}{\partial X^{i}}(0, \xi)=\sum_{n \geq 0}(-1)^{n} \frac{\partial^{i+n} U}{\partial X^{i}}(\lambda, \xi) \lambda^{n}
$$

we have

Similarly

$$
\frac{\partial^{i} U}{\partial X^{i}}(\lambda, \xi)=0 \text { for all } i \geq 0 \quad \Leftrightarrow \quad \frac{\partial^{i} U}{\partial X^{i}}(0, \xi)=0 \text { for all } i \geq 0 .
$$

$$
\begin{array}{lll}
\frac{\partial^{i+1} U}{\partial X^{i} \partial Y}(\lambda, \xi)=0 \quad \text { for all } i \geq 0 & \Leftrightarrow & \frac{\partial^{i+1} U}{\partial X^{i} \partial Y}(0, \xi)=0 \quad \text { for all } i \geq 0, \\
\frac{\partial^{j} U}{\partial Y^{j}}(\lambda, \xi)=0 \text { for all } j \geq 0 & \Leftrightarrow & \frac{\partial^{j} U}{\partial Y^{j}}(\lambda, 0)=0 \text { for all } j \geq 0, \\
\frac{\partial^{1+j} U}{\partial X \partial Y^{j}}(\lambda, \xi)=0 \quad \text { for all } j \geq 0 & \Leftrightarrow & \frac{\partial^{1+j} U}{\partial X \partial Y^{j}}(\lambda, 0)=0 \quad \text { for all } j \geq 0 .
\end{array}
$$

Hence $U(X+\lambda, Y+\xi) \in k[X] \otimes Y^{2} k[Y]$ if and only if

$$
\frac{\partial^{i} U}{\partial X^{i}}(0, \xi)=\frac{\partial^{i+1} U}{\partial X^{i} \partial Y}(0, \xi)=0 \text { for all } i \geq 0,
$$


and that $U(X+\lambda, Y+\xi) \in X^{2} k[X] \otimes k[Y]$ if and only if

$$
\frac{\partial^{j} U}{\partial Y^{j}}(\lambda, 0)=\frac{\partial^{1+j} U}{\partial X \partial Y^{j}}(\lambda, 0)=0 \text { for all } j \geq 0 .
$$

But, it is easy to see that equalities (3.1) are equivalent to conditions in item(1)(a), and equalities (3.2) are equivalent to conditions in item(1)(b). Now, the corollary follows easily from Theorems 3.1 and 3.4 .

Corollary 3.6. Let R. The following facts hold:

(1) If $R \in k \otimes k Y+k[X] \otimes Y^{2} k[Y]$, then there exists a left bounded twisting map $s^{\prime}: k[Y] \otimes k[X] \rightarrow k[X] \otimes k[Y]$ such that $s^{\prime}(Y \otimes X)=R$, if and only if $\frac{\partial R}{\partial Y}(0,0)$ is a multiple root of $\frac{\partial^{j} R}{\partial Y^{j}}(X, 0)$ for each $j>1$.

(2) If $R \in k X \otimes k+X^{2} k[X] \otimes k[Y]$, then there exists a right bounded twisting map $s^{\prime}: k[Y] \otimes k[X] \rightarrow k[X] \otimes k[Y]$ such that $s^{\prime}(Y \otimes X)=R$, if and only if $\frac{\partial R}{\partial X}(0,0)$ is a multiple root of $\frac{\partial^{i} R}{\partial Y^{i}}(X, 0)$ for each $i>1$.

Proof. Item (2) follows immediately from item (1), since $s^{\prime}$ is a twisting map if and only if $\tau \circ s^{\prime} \circ \tau$ is, where $\tau$ is the flip. So, we are reduced to prove the first item. This follows from Corollary 3.5 , since $\left(\frac{\partial R}{\partial Y}(0,0), 0\right)$ always satisfies the conditions asked for $(\lambda, \xi)$ in item (1)(a) of that corollary and $\left(\frac{\partial R}{\partial Y}(0,0), 0\right)$ satisfies those required to $(\lambda, \xi)$ in item (1)(b) if and only if $\frac{\partial R}{\partial Y}(0,0)$ is a multiple root of $\frac{\partial^{j} R}{\partial Y^{j}}(X, 0)$ for each $j>1$.

The Corollary gains in interest if we realize that in item (1) we get all the left bounded twisting maps with $\alpha_{0}=0$ and $\alpha_{1}$ the evaluation in an element of $k$.

\section{NON-COMmUtative EXtensions OF the DUAL NUMBERS}

It seems very difficult to compute all the twisting maps $s: k[Y] \otimes A \rightarrow A \otimes k[Y]$ for a particular algebra $A$. In this section we accomplish this for $A=k[t] /\left\langle t^{2}\right\rangle$ using the evident fact that $s$ is a twisting map if and only if $\tau \circ S_{\circ} \tau$ is also, where $\tau$ denotes the flip.

Theorem 4.1. Let $A$ be an algebra and $s: k[t] /\left\langle t^{2}\right\rangle \otimes A \rightarrow A \otimes k[t] /\left\langle t^{2}\right\rangle$ a twisting map. The maps $\iota_{0}: A \rightarrow A$ and $\iota_{1}: A \rightarrow A$, defined by

$$
s(t \otimes a)=\iota_{0}(a) \otimes 1+\iota_{1}(a) \otimes t,
$$

satisfy:

(1) $\iota_{1}$ is a morphism of algebras.

(2) $\iota_{0}(a b)=\iota_{0}(a) b+\iota_{1}(a) \iota_{0}(b)$ (that is, $\iota_{0}$ is an $\iota_{1}$-derivation).

(3) $\iota_{0}^{2}=0$ and $\iota_{0}^{\circ} \iota_{1}=-\iota_{1}^{\circ} \iota_{0}$.

Conversely, given maps $\iota_{0}: A \rightarrow A$ and $\iota_{1}: A \rightarrow A$ satisfying (1)-(3), the formula (4.1) determines a twisting map.

Proof. Left to the reader.

Lemma 4.2. We define

$$
A_{n}^{N}=\sum_{k=0}^{N-n}(-1)^{k}\left(\begin{array}{c}
n+k \\
n
\end{array}\right)
$$


Then the following facts hold:

$$
\begin{aligned}
& 2 A_{n}^{N}-A_{n-1}^{N-1}=(-1)^{N-n}\left(\begin{array}{c}
N \\
n
\end{array}\right), \\
& 2 A_{n}^{N}-A_{n-1}^{N}=(-1)^{N-n}\left(\begin{array}{c}
N+1 \\
n
\end{array}\right), \\
& 2 A_{0}^{N}=(-1)^{N}+1, \quad A_{N}^{N}=1 .
\end{aligned}
$$

Proof. Assume $N-n=2 j$ is even. Since $\left(\begin{array}{c}n+k \\ n\end{array}\right)-\left(\begin{array}{c}n+k+1 \\ n\end{array}\right)=-\left(\begin{array}{c}n+k \\ n-1\end{array}\right)$,

$$
A_{n}^{N}=-\sum_{k=0}^{j-1}\left(\begin{array}{c}
n+2 k \\
n-1
\end{array}\right)+\left(\begin{array}{c}
N \\
n
\end{array}\right) \quad \text { and } \quad A_{n}^{N}=\sum_{k=0}^{j}\left(\begin{array}{c}
n+2 k-1 \\
n-1
\end{array}\right)
$$

Summing both results, we obtain

$$
2 A_{n}^{N}=\sum_{k=0}^{N-n}(-1)^{k}\left(\begin{array}{c}
n-1+k \\
n-1
\end{array}\right)+(-1)^{N-n}\left(\begin{array}{c}
N \\
n
\end{array}\right)
$$

and so

$$
2 A_{n}^{N}=A_{n-1}^{N-1}+(-1)^{N-n}\left(\begin{array}{c}
N \\
n
\end{array}\right)
$$

which is (4.2). The case $N-n$ odd is similar. The equality (4.3) follows from (4.2). Finally, (4.4) can be easily checked by a direct computation.

Lemma 4.3. A vector $y=\left(y_{i}\right) \in k^{m}$ satisfies the set of equalities

$$
\sum_{i=h}^{m-1} y_{i+1} A_{i-h}^{i}=0 \quad \text { for all } h=0, \ldots, m-1,
$$

where $A_{n}^{N}=\sum_{k=0}^{N-n}(-1)^{k}\left(\begin{array}{c}n+k \\ n\end{array}\right)$, if and only if it satisfies the set of equalities:

$$
\sum_{i=h}^{m}\left(\begin{array}{l}
i \\
h
\end{array}\right) y_{i}=(-1)^{h} y_{h} \quad \text { for all } h=0, \ldots, m .
$$

Proof. Set

$$
A(h)=\sum_{i=h}^{m-1} y_{i+1} A_{i-h}^{i} \quad \text { and } \quad B(h)=\sum_{i=h}^{m}\left(\begin{array}{l}
i \\
h
\end{array}\right) y_{i}-(-1)^{h} y_{h} .
$$

We claim that

$$
2 A(h)-A(h+1)=(-1)^{h} B(h+1) \quad \text { for all } h=0, \ldots, m-1 .
$$

From this equality and from $A(0)=B(0)$ it follows by induction on $h$ that

$$
A(h)=\sum_{k=0}^{h}(-1)^{k} 2^{h-k} B(k),
$$


and hence the lemma. Now we prove the claim:

$$
\begin{aligned}
2 A(h)-A(h+1) & =\sum_{i=h}^{m-1} 2 A_{i-h}^{i} y_{i+1}-\sum_{i=h+1}^{m-1} A_{i-h-1}^{i} y_{i+1} \\
& =\sum_{i=h+1}^{m-1}\left(2 A_{i-h}^{i}-A_{i-h-1}^{i}\right) y_{i+1}+y_{h+1} 2 A_{0}^{h} \\
& =\sum_{i=h+1}^{m-1}(-1)^{h}\left(\begin{array}{c}
i+1 \\
i-h
\end{array}\right) y_{i+1}+y_{h+1}\left((-1)^{h}+1\right) \\
& =\sum_{i=h+1}^{m}(-1)^{h}\left(\begin{array}{c}
i \\
h+1
\end{array}\right) y_{i}+y_{h+1} \\
& =(-1)^{h} B(h+1),
\end{aligned}
$$

where the third equality follows from equalities (4.3) and (4.4).

Theorem 4.4. Let $s: k[t] /\left\langle t^{2}\right\rangle \otimes k[Y] \rightarrow k[Y] \otimes k[t] /\left\langle t^{2}\right\rangle$ be a twisting map. Write $s(t \otimes Y)=Q \otimes 1+P \otimes t$. If $Q \neq 0$, then $P=-Y+p_{0}$ and

(1) If $p_{0}=0$, then $Q \in k\left[Y^{2}\right]$,

(2) If $p_{0} \neq 0$, then $\frac{\partial Q}{\partial Y}\left(p_{0}\right)=(-1)^{i} \frac{\partial Q}{\partial Y}(0)$ for all $i=0, \ldots, m=\operatorname{dg}(Q)$.

(Note that item (2) with $i=m$ implies that $m$ is even). Conversely, if $Q=0$ and $P$ is arbitrary or $P=-Y+p_{0}$ and conditions (1), (2) are satisfied, then there is a unique twisting map $s: k[t] /\left\langle t^{2}\right\rangle \otimes k[Y] \rightarrow k[Y] \otimes k[t] /\left\langle t^{2}\right\rangle$ such that $s(t \otimes Y)=Q \otimes 1+P \otimes t$.

Proof. Let $s$ be a twisting map and $\iota_{j}: k[Y] \rightarrow k[Y](j=0,1)$ be the maps introduced in Theorem 4.1. Assume $Q=\sum_{i=0}^{m} q_{i} Y^{i} \neq 0$. In the sequel we adopt the convention that $P^{0}=1$ even if $P=0$. It is easy to check by induction on $l$, that

$$
\iota_{0}\left(Y^{l}\right)=\sum_{i=0}^{l-1} \iota_{1}(Y)^{i} \iota_{0}(Y) Y^{l-i-1}=Q \sum_{i=0}^{l-1} P^{i} Y^{l-i-1} .
$$

We claim that $\operatorname{dg}(P)=1$. Suppose $\operatorname{dg}(P) \neq 1$ or $P=0$. Let $l \geq 1$. If $\operatorname{dg}(P)>1$, then $\iota_{0}\left(Y^{l}\right)$ has degree $(l-1) \operatorname{dg}(P)+\operatorname{dg}(Q)$, and if $P$ is a constant, then it has degree $l-1+\operatorname{dg}(Q)$. In both cases it is easy to see that $\iota_{0}^{2}=0 \Leftrightarrow \operatorname{dg}(Q)=0$. So, by item (3) of Theorem 4.1, it must be $m=0$. Let $Q=q \in k \backslash\{0\}$. It is immediate that if $\operatorname{dg}(P)>1$, then $\iota_{0}\left(\iota_{1}(Y)\right)=\iota_{0}(P)$ has degree $(\operatorname{dg}(P)-1) \operatorname{dg}(P)$. Since $\iota_{1}\left(\iota_{0}(Y)\right)=q$, it is impossible that $\iota_{0} \iota_{1}=-\iota_{1} \iota_{0} \iota_{0}$. So, again by item $(3)$ of Theorem 4.1, we have $P=0$ or $\operatorname{dg}(P) \leq 1$. If $P$ is a constant, then $\iota_{0}\left(\iota_{1}(Y)\right)=0$ and also in this case $\iota_{0}^{\circ} \iota_{1} \neq-\iota_{1} \iota_{0}$. This proves the claim. Write $P=p_{1} Y+p_{0}$. We assert that the following facts hold

$$
\begin{aligned}
& \text { (3) } \iota_{0}^{2}(Y)=Q \sum_{h=0}^{m-1} \sum_{i=h}^{m-1} \sum_{j=i-h}^{i}\left(\begin{array}{c}
j \\
i-h
\end{array}\right) p_{1}^{j-i+h} p_{0}^{i-h} q_{i+1} Y^{h} . \\
& \text { (4) } \iota_{1}\left(\iota_{0}(Y)\right)=\sum_{i=0}^{m} \sum_{j=i}^{m}\left(\begin{array}{l}
j \\
i
\end{array}\right) q_{j} p_{0}^{j-i} p_{1}^{i} Y^{i} \text { and } \iota_{0}\left(\iota_{1}(Y)\right)=p_{1} \sum_{i=0}^{m} q_{i} Y^{i} .
\end{aligned}
$$

In fact by (4.5), we have

$$
\iota_{0}^{2}(Y)=\sum_{i=0}^{m} q_{i} \iota_{0}\left(Y^{i}\right)=Q \sum_{i=1}^{m} \sum_{j=0}^{i-1} q_{i} P^{j} Y^{i-j-1} .
$$


Since $P^{j}=\sum_{l=0}^{j}\left(\begin{array}{l}j \\ l\end{array}\right) p_{1}^{j-l} p_{0}^{l} Y^{j-l}$, this gives

$$
\iota_{0}^{2}(Y)=Q \sum_{i=1}^{m} \sum_{j=0}^{i-1} \sum_{l=0}^{j} q_{i}\left(\begin{array}{l}
j \\
l
\end{array}\right) p_{1}^{j-l} p_{0}^{l} Y^{i-l-1} .
$$

A change of indices yields item (3). Next we check item (4). We have

$$
\iota_{1}\left(\iota_{0}(Y)\right)=\sum_{j=0}^{m} q_{j} \iota_{1}(Y)^{j}=\sum_{j=0}^{m} \sum_{l=0}^{j} q_{j}\left(\begin{array}{l}
j \\
l
\end{array}\right) p_{1}^{j-l} p_{0}^{l} Y^{j-l}=\sum_{j=0}^{m} \sum_{i=0}^{j} q_{j}\left(\begin{array}{l}
j \\
i
\end{array}\right) p_{1}^{i} p_{0}^{j-1} Y^{i} .
$$

Interchanging the sums we obtain item (4), since the second equality is clear. Considering now the terms of maximal degree in items (3) and (4), we get

$$
\sum_{j=0}^{m-1} p_{1}^{j}=0 \quad \text { and } \quad p_{1}^{m}=-p_{1}
$$

since, by Theorem 4.1 we know that

$$
\iota_{0}^{2}(Y)=0 \quad \text { and } \quad \iota_{1}\left(\iota_{0}(Y)\right)=-\iota_{0}\left(\iota_{1}(Y)\right)
$$

Hence $p_{1}=-1$ and equalities (3) and (4) become

(5) $\iota_{0}^{2}(Y)=Q \sum_{h=0}^{m-1} \sum_{i=h}^{m-1} \sum_{j=i-h}^{i}\left(\begin{array}{c}j \\ i-h\end{array}\right)(-1)^{j-i+h} p_{0}^{i-h} q_{i+1} Y^{h}$

(6) $\iota_{1}\left(\iota_{0}(Y)\right)=\sum_{i=0}^{m} \sum_{j=i}^{m}\left(\begin{array}{l}j \\ i\end{array}\right) q_{j} p_{0}^{j-i}(-1)^{i} Y^{i}$ and $\iota_{0}\left(\iota_{1}(Y)\right)=-\sum_{i=0}^{m} q_{i} Y^{i}$.

From this it follows immediately that equalities (4.6) implies items (1) and (2). Now we prove the second part. First note that for $P, Q \in k[Y]$ arbitrary, equalities $\iota_{0}(Y)=Q$ and $\iota_{1}(Y)=P$ determine unique maps $\iota_{0}, \iota_{1}: k[Y] \rightarrow k[Y]$ satisfying conditions (1) and (2) of Theorem 4.1. Clearly condition (3) is also fulfilled if and only if $\iota_{0}^{2}(Y)=0$ and $\iota_{1}\left(\iota_{0}(Y)\right)=-\iota_{0}\left(\iota_{1}(Y)\right)$. When $Q=0$ these last equalities are trivially true. Assume now $Q \neq 0$ and $P=-Y+p_{0}$. Then, arguing as above, we obtain that (5) and (6) are satisfied. From this it follows immediately that, if $p_{0}=0$ and $q_{i}=0$ for $i$ odd, then equalities (4.6) are true. Assume now that $p_{0} \neq 0$. In this case from (5) and (6) it follows that $\iota_{0}^{2}(Y)=0$ if and only if the $q_{i}$ 's satisfy

$$
\sum_{i=h}^{m-1} q_{i+1} p_{0}^{i+1} A_{i-h}^{i}=0 \quad \text { for all } h=0, \ldots, m-1,
$$

where $A_{n}^{N}:=\sum_{k=0}^{N-n}(-1)^{k}\left(\begin{array}{c}n+k \\ n\end{array}\right)$ and that $\iota_{1}\left(\iota_{0}(Y)\right)=-\iota_{0}\left(\iota_{1}(Y)\right)$ if and only if the $q_{i}$ 's satisfy

$$
\sum_{j=i}^{m}\left(\begin{array}{l}
j \\
i
\end{array}\right) q_{j} p_{0}^{j-i}=(-1)^{i} q_{i} \quad \text { for all } i=0, \ldots, m
$$

which is true by item (2). But, by Lemma 4.3, applied to $\left\{y_{i}=q_{i} p_{0}^{i}\right\}$, the last set of equalities implies the first one. So the theorem is proved.

In the previous theorem we found necessary and sufficient conditions, on polynomials $P, Q \in k[Y]$, in order that a twisting map

$$
s: k[t] /\left\langle t^{2}\right\rangle \otimes k[Y] \rightarrow k[Y] \otimes k[t] /\left\langle t^{2}\right\rangle
$$

such that $s(t \otimes Y)=P \otimes t+Q \otimes 1$ exists. If $Q=0$, then $P$ is arbitrary and if $Q \neq 0$ then $P=-Y+p_{0}$ and items (1) or (2) of Theorem 4.4 must be satisfied, depending on if $p_{0}=0$ or $p_{0} \neq 0$. In the first case the condition is simply that $Q \in k\left[Y^{2}\right]$. The second case is more involved and we give a complete solution under the hypothesis that $k$ is a characteristic zero field. 
Corollary 4.5. (Classification of the non-commutative extension of the algebra of dual numbers by $k[Y])$. Let $k$ be a characteristic zero field. If $Q \neq 0$, then any choice of $p_{0} \in k \backslash\{0\}, m$ even and $q_{0}, q_{2}, q_{4}, \ldots, q_{m-2}, q_{m} \in k$, with $q_{m} \neq 0$ determines univocally polynomials $P=-Y+p_{0}$ and $Q=\sum_{i=0}^{m} q_{i} Y^{i}$ satisfying condition (2) of Theorem 4.4.

Proof. For each $i \geq 0$, let $y_{i}=q_{i} p_{0}^{i}$. Consider $\mathbf{y}=\left(y_{0}, \ldots, y_{m}\right)$ as a column vector. By item (3) of Theorem 4.4, there is a twisting map

$$
s: k[t] /\left\langle t^{2}\right\rangle \otimes k[Y] \rightarrow k[Y] \otimes k[t] /\left\langle t^{2}\right\rangle
$$

such that $s(t \otimes Y)=P \otimes t+Q \otimes 1$, if and only if $\sum_{j=i}^{m}\left(\begin{array}{l}j \\ i\end{array}\right) y_{j}-(-1)^{i} y_{i}=0$ for $i=0, \ldots, m$. Write the system of equations

$$
B(i)=\sum_{j=i}^{m}\left(\begin{array}{l}
j \\
i
\end{array}\right) y_{j}-(-1)^{i} y_{i}=0 \quad(j=0, \ldots, m)
$$

in the matrix form $\mathbf{C y}=\mathbf{0}$. It is then easy to see that $\mathbf{C}=\left(c_{i j}\right)_{0 \leq i, j \leq m}$ is

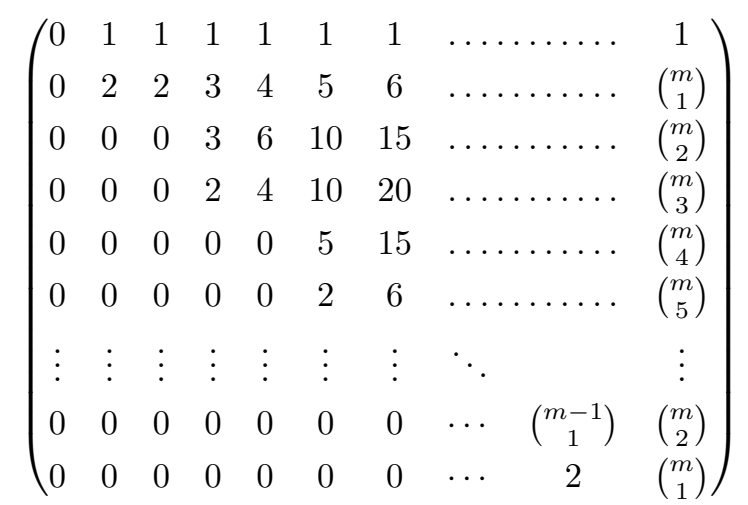

By the shape of this matrix it is clear that the even rows are linearly independent and so we only need to prove that $\operatorname{rank}(\mathbf{C})=m / 2$. For this it suffices to check that

$$
\sum_{k=0}^{n}(-1)^{k} c_{i, 2 n-k}\left(\begin{array}{l}
n \\
k
\end{array}\right)=0 \quad \text { for } n \geq 1 \text { and } i=0, \ldots, m,
$$

since then the even columns will be linear combinations of the previous ones. Let

$$
D_{i j}^{(n)}=\left\{\begin{array}{ll}
\left(\begin{array}{c}
j-n \\
i-n
\end{array}\right) & \text { if } n \leq i \leq j, \\
0 & \text { otherwise }
\end{array} \quad \text { and } \quad E_{i j}^{(n)}= \begin{cases}(-1)^{j+1}\left(\begin{array}{c}
n \\
j-i
\end{array}\right) & \text { if } j-n \leq i \leq j \\
0 & \text { otherwise }\end{cases}\right.
$$

Since $E_{i, 2 n}^{(n)}+D_{i, 2 n}^{(n)}=0$ in order to prove (4.7) it is enough to show that

$$
E_{i j}^{(n)}+D_{i j}^{(n)}=\sum_{k=0}^{n}\left(\begin{array}{l}
n \\
k
\end{array}\right)(-1)^{k} c_{i, j-k} \quad \text { for } i=0, \ldots, m \text { and } j \geq n .
$$

This follows immediately from the equalities

$$
\begin{aligned}
& E_{i j}^{(0)}+D_{i j}^{(0)}=c_{i j}, \\
& D_{i j}^{(n)}=\sum_{k=0}^{n}(-1)^{k} D_{i, j-k}^{(0)}\left(\begin{array}{l}
n \\
k
\end{array}\right), \\
& E_{h j}^{(n)}=\sum_{k=0}^{n}(-1)^{k} E_{i, j-k}^{(0)}\left(\begin{array}{l}
n \\
k
\end{array}\right),
\end{aligned}
$$

for $i=0, \ldots, m$ and $j \geq n$. The first and the third one can be checked by a direct computation, while the second one by induction on $n$. 
Let $s, P$ and $Q$ be as in Theorem 4.4. Let $\alpha_{j}: k[t] /\left\langle t^{2}\right\rangle \rightarrow k[t] /\left\langle t^{2}\right\rangle$ be the maps defined by

$$
\tau \circ S^{\circ} \tau(Y \otimes t)=\sum_{j=0}^{\infty} \alpha_{j}(t) \otimes Y^{j},
$$

where $\tau$ is the flip. If $Q=0$ and $P=\sum_{i=0}^{n} p_{i} Y^{i}$, then $\alpha_{j}(t)=p_{j} t$. If $P$ and $Q$ are as in items (1) and (2) of Theorems 4.4, then

$$
\alpha_{j}(t)= \begin{cases}q_{0}+p_{0} t & \text { if } j=0, \\ q_{1}-t & \text { if } j=1, \\ q_{j} & \text { if } 2 \leq j \leq m, \\ 0 & \text { if } j>m .\end{cases}
$$

\section{TWISTED EXTENSIONS BY POWER SERIES}

Let $k$ be a commutative ring. This section is devoted to the study of twisting tensor products between the power series ring $k[[Y]]$ and a filtered complete algebra $A$. Hence we work in the monoidal category CMod of complete filtered $k$-modules (see Section 1). Recall that the tensor product of CMod is denoted by $\hat{\otimes}$. We will use freely the notations introduced in Sections 1 and 2.

Lemma 5.1. Let $A$ be a filtered complete algebra and $\left(\alpha_{j}: A \rightarrow A\right)_{j \geq 0}$ a family of continuous maps. If for each $i \geq 0$ there exists $n_{0} \geq 0$ such that $\alpha_{0}^{n}(\bar{A}) \subseteq A_{i}$ for all $n \geq n_{0}$, then for each $i, j \geq 0$ there exists $r_{0} \geq 0$ such that $\gamma_{j}^{(r)}\left(A_{h}\right) \subseteq A_{i}$ whenever $r+h \geq r_{0}$.

Proof. We proceed by induction on $j$. First we assume $j=0$. By hypothesis there exists $n_{0} \geq 0$, such that $\gamma_{0}^{(n)}(A)=\alpha_{0}^{n}(A) \subseteq A_{i}$ whenever $n \geq n_{0}$. Since $\alpha_{0}$ is continuous, there exists $h_{0} \geq 0$ such that $\gamma_{0}^{(n)}\left(A_{h}\right)=\alpha_{0}^{n}\left(A_{h}\right) \subseteq A_{i}$, for each $n<n_{0}$ and $h>h_{0}$. Clearly we can take $r_{0}=n_{0}+h_{0}$. Assume the lemma is valid for $j$ and write

$$
\gamma_{j+1}^{(r)}=\sum_{l_{1}=0}^{r-1} \sum_{l_{2}=1}^{j+1} \alpha_{0}^{l_{1}}{ }^{\circ} \alpha_{l_{2}} \circ \gamma_{j+1-l_{2}}^{\left(r-l_{1}-1\right)} .
$$

Since $\alpha_{0}^{n}(A) \subseteq A_{i}$ for all $n \geq n_{0}$ in order to complete the inductive step it suffices to show that for all $l_{1}<n_{0}$ and $l_{2} \leq j+1$, there exists $r_{0} \geq 0$ such that

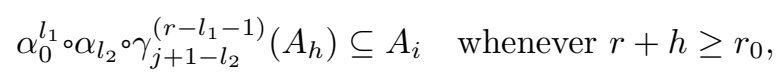

which follows immediately from the continuity of $\alpha_{0}^{l_{1}}{ }^{\circ} \alpha_{l_{2}}$ and the inductive hypothesis.

Remark 5.2. If $s: k[[Y]] \hat{\otimes} A \rightarrow A \hat{\otimes} k[[Y]]$ is a twisting map, then $\operatorname{ker}\left(\alpha_{0}\right)$ is a closed subalgebra of $A$. Moreover,

- If $\alpha_{0}=0$, then $\alpha_{1}$ is a endomorphism of algebras.

- Let $\nu>1$. If $\alpha_{j}=0$ for $1<j<\nu$, then

$$
\alpha_{\nu}(a b)=\alpha_{1}(a) \alpha_{\nu}(b)+\alpha_{\nu}(a) \alpha_{1}^{\nu}(b) .
$$

Theorem 5.3. Let $A$ be a filtered complete algebra and $s: k[[Y]] \hat{\otimes} A \rightarrow A \hat{\otimes} k[[Y]]$ a twisting map. The equation

$$
s(Y \hat{\otimes} a)=\sum_{j=0}^{\infty} \alpha_{j}(a) \hat{\otimes} Y^{j},
$$

defines a family of maps $\alpha_{j}: A \rightarrow A$, which satisfies: 
(1) The $\alpha_{j}$ 's are continuous maps.

(2) For each $i \geq 0$ there exists $n_{0}$ such that $\alpha_{0}^{n}(A) \subseteq A_{i}$ for all $n \geq n_{0}$.

(3) $\alpha_{j}(1)=\delta_{j 1}$.

(4) For all $j \geq 0$ and all $a, b \in A$,

$$
\alpha_{j}(a b)=\sum_{r=0}^{\infty} \alpha_{r}(a) \gamma_{j}^{(r)}(b) \quad \text { (this formula makes sense by Lemma 5.1). }
$$

Moreover,

$$
s\left(\sum_{r=0}^{\infty} Y^{r} \hat{\otimes} a_{r}\right)=\sum_{j=0}^{\infty}\left(\sum_{r=0}^{\infty} \gamma_{j}^{(r)}\left(a_{r}\right)\right) \hat{\otimes} Y^{j} .
$$

Conversely, given maps $\alpha_{j}: A \rightarrow A$ (j $\geq 0$ ) satisfying (1)-(4), the formula (5.1) defines a twisting map.

Proof. Items (1) and (2) follows easily from the continuity of $s$, and items (3) and (4) can be checked as in the proof of Theorem 2.1. To check (5.1) we can assume that only one $a_{r} \neq 0$. In this case we can proceed again as in the proof of Theorem 2.1. Conversely, assume we have a family of continuous maps $\left(\alpha_{j}\right)_{j \geq 0}$ satisfying (1), (2), (3) and (4) and define $s$ by the formula (5.1). By Lemma 5.1 this map is well defined and it is continuous. We leave the task to prove that $s$ is a twisting map to the reader.

Theorem 5.4. Let $\alpha: A \rightarrow A$ be an automorphism of filtered completed algebras. For each $i \geq 1$, let $\beta_{i}: A \rightarrow A$ be a continuous $\left(\alpha, \alpha^{i+1}\right)$-derivation. If

$$
\alpha^{r}\left(\beta_{i}(a)\right) \beta_{i^{\prime}}(b)=0 \quad \text { for all } r \in \mathbb{Z} \text { and } a, b \in A \text { whenever } i+i^{\prime} \geq 3,
$$

then, the formula

$$
s(Y \otimes a)=\sum_{j=0}^{\infty} \alpha_{j}(a) \otimes Y^{j},
$$

where the maps $\alpha_{j}: A \rightarrow A(j \geq 0)$ are constructed as in Lemma 2.5, defines a twisting map $s: k[[Y]] \hat{\otimes} A \rightarrow A \hat{\otimes} k[[Y]]$.

Proof. Mimic the proof of Theorem 2.7.

Lemma 5.5. Let $Q \in k[[X]] \hat{\otimes} Y k[[Y]]$. The equality $\alpha_{1}(X)=\frac{\partial Q}{\partial Y}(X, 0)$ defines a continuous algebra map $\alpha_{1}: k[[X]] \rightarrow k[[X]]$ if and only if the independent term $\frac{\partial Q}{\partial Y}(0,0)$ of $\alpha_{1}(X)$ is nilpotent. Moreover, in this case, there is a unique family of continuous maps $\left(\alpha_{j}: k[[X]] \rightarrow k[[X]]\right)_{j \geq 2}$, that satisfy

$$
\begin{aligned}
& \alpha_{j+1}(1)=\delta_{j+1,1}, \\
& \alpha_{j+1}(X)=\frac{1}{(j+1) !} \frac{\partial^{j+1} Q}{\partial Y^{j+1}}(X, 0), \\
& \alpha_{j+1}\left(X^{n+1}\right)=\sum_{r=1}^{j+1} \alpha_{r}\left(X^{n}\right) \gamma_{j+1}^{(r)}(X),
\end{aligned}
$$

for all $j \geq 1$.

Proof. The first assertion is immediate. Let $q=\frac{\partial Q}{\partial Y}(0,0)$. In order to prove the second one it will be sufficient to show that

$$
\alpha_{j}\left(X^{n}\right) \in \sum_{r=0}^{n-j+1} q^{n-j-r+1} X^{r} k[[X]] \quad \text { if } n \geq j .
$$


We will prove this fact by induction on $j$. For $j=1$ this is clear. Assume it is true for $j$ and for $\alpha_{j+1}\left(X^{h}\right)$ with $j \leq h \leq n$. Then, by the inductive hypothesis and the facts that

$$
\alpha_{j+1}\left(X^{n+1}\right)=\sum_{s=1}^{j+1} \alpha_{s}\left(X^{n}\right) \gamma_{j+1}^{(s)}(X)
$$

and

$$
\gamma_{j+1}^{(j+1)}(X)=\alpha_{1}^{j+1}(X) \in q k[[X]]+X k[[X]],
$$

we get that for all $n \geq j$,

$$
\alpha_{j+1}\left(X^{n+1}\right) \in \sum_{s=1}^{j} \sum_{r=0}^{n-s+1} q^{n-s-r+1} X^{r} k[[X]]+q^{n-j-r} X^{r} k[[X]](q k[[X]]+X k[[X]]) .
$$

Using this the proof can be easily finished.

Theorem 5.6. Let $Q \in k[[X]] \hat{\otimes} Y k[[Y]]$. If $\frac{\partial Q}{\partial Y}(0,0)$ is nilpotent, then there is a unique twisting map $s: k[[Y]] \hat{\otimes} k[[X]] \rightarrow k[[X]] \hat{\otimes} k[[Y]]$ such that $s(Y \hat{\otimes} X)=Q$. Moreover

$$
s(Y \hat{\otimes} P)=\sum_{j \geq 1} \alpha_{j}(P) \hat{\otimes} Y^{j},
$$

where $\alpha_{j}: k[[X]] \rightarrow k[[X]](j \geq 1)$ are the maps introduced in Lemma 5.5.

Proof. The uniqueness and the last assertion are immediate. Let us prove the existence. Let $\alpha_{0}=0$. By Lemma 5.5 we know that the maps $\alpha_{j}$ are well defined and continuous. Moreover, it is evident that items (1), (2) and (3) of Theorem 5.3 are satisfied. So we only must prove item (4), which (by linearity and continuity) reduce to check that

$$
\alpha_{j}\left(X^{m} X^{n}\right)=\sum_{l=1}^{j} \alpha_{l}\left(X^{m}\right) \gamma_{j}^{(l)}\left(X^{n}\right) \quad \text { for all } m, n \geq 0 .
$$

For $j=1$ this follows from Remark 5.2. Assume that the result is true for $\alpha_{i}$ with $i<j$ and for $\alpha_{j}\left(X^{m} X^{v}\right)$ with $v \leq n$. By the recursive definition of $\alpha_{j}$ and the inductive hypothesis,

$$
\begin{aligned}
\alpha_{j}\left(X^{m} X^{n+1}\right) & =\sum_{r=1}^{j} \alpha_{r}\left(X^{m} X^{n}\right) \gamma_{j}^{(r)}(X) \\
& =\sum_{r=1}^{j} \sum_{l=1}^{r} \alpha_{l}\left(X^{m}\right) \gamma_{r}^{(l)}\left(X^{n}\right) \gamma_{j}^{(r)}(X) \\
& =\sum_{l=1}^{j} \sum_{r=l}^{j} \alpha_{l}\left(X^{m}\right) \gamma_{r}^{(l)}\left(X^{n}\right) \gamma_{j}^{(r)}(X) .
\end{aligned}
$$

So it is enough to show that

$$
\gamma_{j}^{(l)}\left(X^{n+1}\right)=\sum_{r=l}^{j} \gamma_{r}^{(l)}\left(X^{n}\right) \gamma_{j}^{(r)}(X) \quad \text { for } l=1, \ldots, j .
$$


We prove this formula by induction on $l$. When $l=1$ this is true by the recursive definition of $\alpha_{j}\left(X^{n+1}\right)=\gamma_{j}^{(1)}\left(X^{n+1}\right)$. Suppose $l>1$. Then, we have

$$
\begin{aligned}
\gamma_{j}^{(l)}\left(X^{n+1}\right) & =\sum_{i_{1}=1}^{j-l+1} \sum_{\left|i_{2}, \ldots, i_{l}\right|=j-i_{1}} \alpha_{i_{1}}\left(\alpha_{i_{2} \ldots i_{l}}\left(X^{n+1}\right)\right) \\
& =\sum_{i=1}^{j-l+1} \alpha_{i}\left(\gamma_{j-i}^{(l-1)}\left(X^{n+1}\right)\right) \\
& =\sum_{i=1}^{j-l+1} \sum_{s=l-1}^{j-i} \alpha_{i}\left(\gamma_{s}^{(l-1)}\left(X^{n}\right) \gamma_{j-i}^{(s)}(X)\right) \\
& =\sum_{s=l-1}^{j-1} \sum_{i=1}^{j-s} \sum_{h=1}^{i} \alpha_{h}\left(\gamma_{s}^{(l-1)}\left(X^{n}\right)\right) \gamma_{i}^{(h)}\left(\gamma_{j-i}^{(s)}(X)\right) \\
& =\sum_{s=l-1}^{j-1} \sum_{h=1}^{j-s} \sum_{i=h}^{j-s} \alpha_{h}\left(\gamma_{s}^{(l-1)}\left(X^{n}\right)\right) \gamma_{i}^{(h)}\left(\gamma_{j-i}^{(s)}(X)\right) \\
& =\sum_{r=l}^{j} \gamma_{r}^{(l)}\left(X^{n}\right) \gamma_{j}^{(r)}(X),
\end{aligned}
$$

as desired.

Remark 5.7. Let $Q=\sum_{i j} q_{i j} X^{i} \hat{\otimes} Y^{j} \in X k[[X]] \hat{\otimes} k[[Y]]$. Suppose $q_{10}=\frac{\partial Q}{\partial X}(0,0)$ is nilpotent. By Theorem 5.6 we know that there exists a unique twisting map $s: k[[X]] \hat{\otimes} k[[Y]] \rightarrow k[[Y]] \hat{\otimes} k[[X]]$ satisfying

$$
s(X \hat{\otimes} Y)=\sum_{i j} q_{i j} Y^{j} \hat{\otimes} X^{i} .
$$

But then the map $\tau \circ S_{\circ} \tau$, where $\tau: k[[Y]] \hat{\otimes} k[[X]] \rightarrow k[[X]] \hat{\otimes} k[[Y]]$ is the flip, is a twisting map taking $Y \otimes X$ to $Q$.

\section{Appendix A}

This appendix is devoted to prove Theorem 3.4. So, we assume that $k$ is a commutative domain. Let $R=k[X] \otimes_{s} k[Y]$ be the twisted algebra determined by $s$. By a sake of simplicity we will write $X$ instead of $X \otimes 1$ and $Y$ instead of $1 \otimes Y$. Hence $R=k[X, Y]$ as a left $k$-module.

In the setting of Theorem 3.4 (1) we have:

Lemma A.1. $R Y$ is a two-sided ideal of $R$, thus $\left(R Y^{m}\right)\left(R Y^{n}\right)=R Y^{m+n}$ for any nonnegative integers $m$ and $n$.

Proof. We have by assumption that $Y X \subseteq R Y$, thus $Y R \subseteq R Y$. The lemma follows.

Lemma A.2. $Y X^{n} \in R Y^{n+1}$ for all $n \geq 1$.

Proof. For $n=1$ we have by assumption $Y X \in R Y^{2}$. We complete the proof by induction on $n$, using Lemma A.1.

Lemma A.3. $Y X^{n}=0$ for $n \gg 0$.

Proof. By the previous lemma, we have $Y X^{n} \in R Y^{n+1}$ for all $n \geq 1$. Since the map $s$ is left bounded, we obtain that $Y X^{n}=0$ for $n \gg 0$.

By assumption we have $Y X=\left(f_{0}+X f_{1}\right) Y^{2}$, where $f_{0} \in k[Y]$ and $f_{1} \in R$. 
Lemma A.4. Suppose that $f_{0} \neq 0$ and write $f_{0}=\bar{f}_{0} Y^{t}$ with $\bar{f}_{0}(0) \neq 0$. Then,

$$
Y^{n} X \in f_{0} Y^{n+1}+R Y^{t+n+2}+X R \text { for all } n \geq 1 \text {. }
$$

Proof. We proceed by induction on $n$. Case $n=1$ is trivial. Assume that the result is valid for $n$. Then, by the inductive hypothesis and Lemma A.1,

$$
\begin{aligned}
Y^{n+1} X & =Y^{n}\left(f_{0}+X f_{1}\right) Y^{2} \\
& =f_{0} Y^{n+2}+Y^{n} X f_{1} Y^{2} \\
& \in f_{0} Y^{n+2}+\left(f_{0} Y^{n+1}+R Y^{t+n+2}+X R\right) f_{1} Y^{2} \\
& \subseteq f_{0} Y^{n+2}+R Y^{t+n+3}+X R,
\end{aligned}
$$

as we want.

Corollary A.5. Assume that $Y X \notin X R$. If $h$ is an element in $R \backslash(k+X R)$, then $h X^{m} \notin k+X R$ for all $m \geq 1$.

Proof. Clearly it is enough to consider the case $m=1$. By assumption $h \in g+X R$, where $g \in k[Y] \backslash k$. Write $g=c+\bar{g} Y^{n}$ with $c \in k$ and $\bar{g}(0) \neq 0$. By the previous lemma and using the same notation

$$
g X=c X+\bar{g} Y^{n} X \in \bar{g} f_{0} Y^{n+1}+R Y^{t+n+2}+X R .
$$

Hence, $g X \notin k+X R$ and so $h X \notin k+X R$.

Lemma A.6. $Y X \in X R$.

Proof. Assume $Y X \notin X R$. Since $Y X \in R Y$, this implies that $Y X \notin k+X R$. So, by the previous corollary, $Y X^{m} \neq 0$ for all $m \geq 1$, contradicting Lemma A.3.

Corollary A.7. $R X \subseteq X R$, so $X R$ is a two-sided ideal of $R$.

Lemma A.8. Assume that $Y X=X g+X^{2} h$, where $g \in k[Y]$ and $h \in R$. Let $f$ be a polynomial in $k[Y]$. Then $f X \in X(f \circ g)+X^{2} R$.

Proof. By the previous corollary, $X^{2} R$ is an ideal of $R$. By induction on $n$ we obtain that $Y^{n} X \equiv X g^{n}\left(\bmod X^{2} R\right)$ for $n \geq 1$. The lemma follows.

We now complete the proof of Theorem 3.4 (1) in the paper:

Theorem A.9. $Y X \in X^{2} R$.

Proof. Assume that $Y X \notin X^{2} R$. We prove by induction that

$$
Y X^{n}=X^{n} g_{n}+X^{n+1} h_{n}
$$

for a nonzero polynomial $g_{n} \in k[Y]$ and an element $h_{n} \in R$. The case $n=1$ holds by assumption and by Lemma A.6. Let $n>1$. By the inductive assumption we have $Y X^{n+1}=\left(Y X^{n}\right) X=\left(X^{n} g_{n}+X^{n+1} h_{n}\right) X$. By Lemma A.8,

$$
g_{n} X=X\left(g_{n} \circ g_{1}\right) \quad\left(\bmod X^{2} R\right) \text {. }
$$

We have $g_{n}^{\circ} g_{1} \neq 0$ since $k$ is an integral domain. Also $h_{n} X \in X R$ by Corollary A.7. Hence $Y X^{n+1}$ is of the desired form. It follows that $Y X^{n} \neq 0$ for all $n$, in contradiction to Lemma A.3. The theorem follows.

Remark A.10. Theorem 3.4 remains valid if we replace the hypothesis that $s$ is left bounded by the weaker one that there exists $n \in \mathbb{N}$ such that

$$
s\left(Y \otimes X^{n}\right) \in k[X] \otimes \bigoplus_{i=0}^{n} k Y^{i} .
$$

Remark A.11. Setting $Y X=f Y$, we have $Y X^{n}=f^{n} Y$ for all $n \geq 0$. This fact was not used in the proof. 


\section{REFERENCES}

[C-S-V] A. Cap, H. Schichl and J. Vanz̃ura On twisted tensor products of algebras, Communications in Algebra, Vol 23 (1995) 4701-4735.

[Ca] P. Cartier Produits tensorieles tordus, Exposé au Séminaire des groupes quantiques de'l École Normale Supérieure, Paris (1991-1992).

[C-I-M-Z] S. Cænepel, B. Ion, G. Militarú, S. Zhu The factorisation problem and smah biproducts of algebras an coalgebras, Algebr. Represent. Theory, Vol 3 (2000) 14-42.

[G-G] J. A. Guccione and J. J. Guccione Hochschild Homology ofTwisted Tensor Products, K-Theory, vol 18 (4) (1999) 363-400.

[Ka] C. Kassel Quantum groups, Graduate Texts in Mathematics, Vol 155 Springer-Verlag, New Xork (1995).

[Ma] S. Majid Algebras and Hopf algebras in braided categories, Advances in Hopf algebras, Marcel Dekker (1993).

[Mo] S. Montgomery Hopf algebras and their actions on rings, CBMS Regional Conference Series in Mathematics, vol 82 AMS Providence Rhode Island (1993).

[Tam] D. Tambara The coendomorphism bialgebra of an algebra, J. Fac Sci. Univ. Tokio Sect. IA Math, vol 34 (1990) 425-456.

[VD-VK] A. Van Daele and S. Van Keer The Xang Baxter and the pentagon equation, Compositio Math., Vol 91 (1994) 201-221.

Departamento de Matemática, Facultad de Ciencias Exactas y Naturales, Universidad de Buenos Ares, Pabellón 1 - Ciudad Universitaria, (1428) Buenos Aires, Argentina.

E-mail address: vander@dm.uba.ar

Departamento de Matemática, Facultad de Ciencias Exactas y Naturales,, Universidad de Buenos Ares, Pabellón 1 - Ciudad Universitaria, (1428) Buenos Aires, Argentina. E-mail address: jjgucci@dm.uba.ar

Pontificia Universidad Católica del Perú - Instituto de Matemática y Ciencias Afines, Sección Matemáticas, PUCP, Av. Universitaria 1801, San Miguel, Lima 32, Perú.

E-mail address: cvalqui@pucp.edu.pe 